\title{
Multi-function Monitoring and Alarm System for the Large Stadium
}

\author{
Wang Xiaohui \\ School of Leisure Management \\ Xi'an Eurasia University \\ Xi'an, China \\ e-mail: wangxiaohui@eurasia.edu
}

\author{
Dou Xiaoning \\ School of Leisure Management \\ Xi'an Eurasia University \\ Xi'an, China \\ e-mail: douxiaoning@eurasia.edu
}

\begin{abstract}
In the recent years, the safety problem in the large stadium has concerned the researchers. Especially during during a big sport event, the safety problem of the stadium has become an important public event. This paper designs a multifunction monitoring and alarm system based on the mobile communication network. It could realize the smoke or combustible gas alarm, the infrared identification detection and the temperature alarm. It collects the data through the sensor and sends the data to the mobile phone or the control platform. of being simple, convenient, save, various functions and low cost.
\end{abstract} GSM

Keywords-Sensor; Wireless Transmission; Alarm; MCU;

\section{INTRODUCTION}

For the safety problem of the large sports venues, this paper introduces a multi-function alarm system based on the wireless communication system[1]. It can realize the stadium smoke or combustible gas, the infrared detection and the temperature alarm. Through the sensor and the wireless communication module, the collected data was send to the user or the main control platform. The system includes the minimum system based on STC89C52, sensor circuit composed of the DS18B20, HC-SR501, MQ-2, GSM communication module and the LCD1602 display circuit. The software system includes the main program, the GSM SMS transceiver program, the display program and the temperature detection program[2].

\section{OVERALL DESIGN OF THE SYSTEM}

\section{A. The system function block}

The hardware system is composed by the smallest system of MCU, the GSM module, the display circuit, the buzzer control circuit and the sensor circuit[3]. The sensor circuit is composed of three parts: the smoke detection, the human infrared detection and the temperature detection. It is showed in Figure 1.

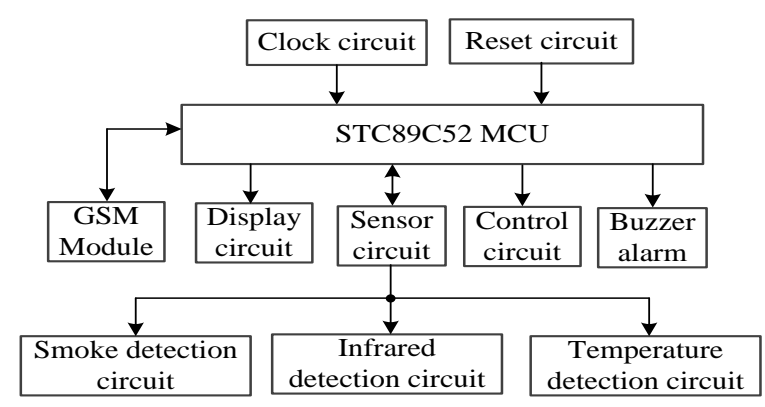

Figure 1. System block diagram.

From Figure 1, when the three sensors detect the smoke, the human body or the high temperature, the alarm information is sent to the microcontroller. The microcontroller detects the alarm by judging the threshold of the buzzer circuit. Then the GSM module sends the alarm message to the user.

\section{B. The design of the minimum system}

The minimum system of single chip microcomputer is composed of single chip microcomputer, crystal oscillator circuit and reset circuit[4]. As shown in Figure 2.

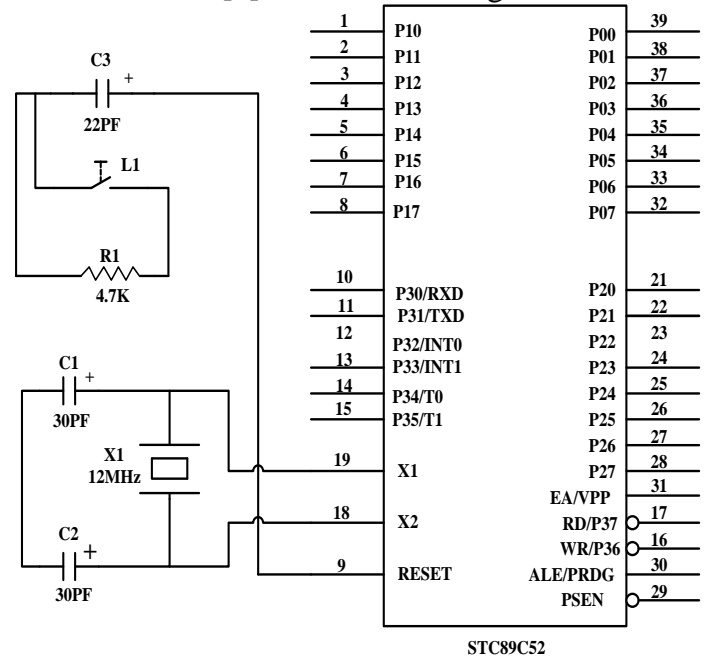

Figure 2. Minimum system of the single chip.

The principle of reset circuit is to reset the program manually when pressing the button, reset the program, and 
restart it. In the crystal oscillator circuit, X1 provides oscillating signal for the crystal oscillator to the microcontroller, and the MCU runs into the running program.

\section{Example Sensor detection circuit}

\section{1) Temperature detection.}

The temperature of the environment is collected by DS18B20 and the collected information is sent to the circuit through the interface. The temperature range is $-55^{\circ} \mathrm{C}$ $\sim+125^{\circ} \mathrm{C}$. The increment is $0.5^{\circ} \mathrm{C}$ and the temperature can be turned into a number. The MCU can read the temperature directly. This temperature sensor reduces the external circuit and makes it easier to realize[5].

The DS18B20 consists of four parts: the 64 bits ROM with the single-wire interface, the temperature sensor, the temperature trigger and the configuration register. Its structure is shown in Figure 3.

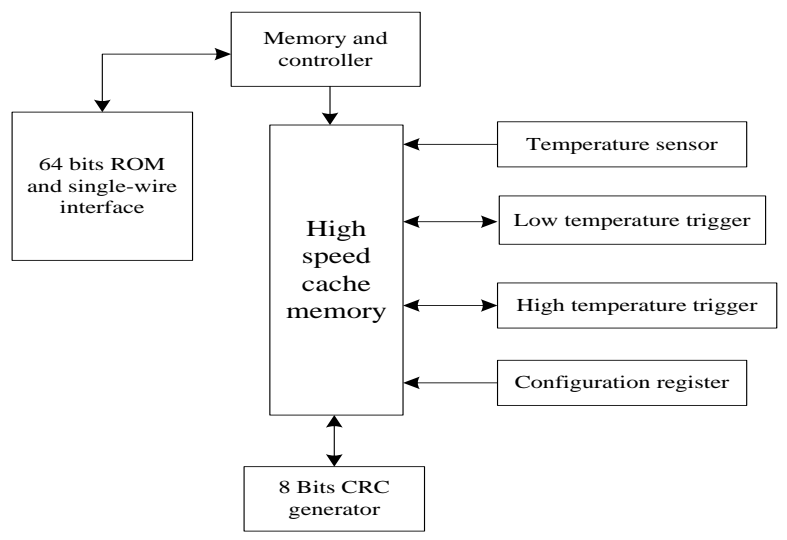

Figure 3. The internal block of DS18B20.

Through the single bus protocol, the single chip microcontroller reads the data and operations from the DS18B20. Finally it is concluded the temperature value. The resistor in the circuit used for the pull-up, it has the ability of the anti-interference.

\section{2) Smoke and combustible gas detection.}

The MQ-2 smoke sensor is a kind of the air sensitive material with the tin oxide. It has the low conductivity in the clean air[6]. Therefore, in the surrounding environment, the conductivity of the sensor increases with the increase in the presence of the smoke or the combustible gas.

LM393 is the operational amplifier which is used as a voltage comparator. When the input voltage $\mathrm{V}+>\mathrm{V}$-, the output is high and the output is low when $\mathrm{V}->\mathrm{V}+$.

The MQ-2 is the smoke sensor. In MQ-2, R1 and RV2 constitute the circuit. RV1 is a sliding resistor which adjusts the input voltage of LM393. In general, when $\mathrm{V}+>\mathrm{V}-$, outputs the high level. When the MQ-2 detects the smoke or the combustible gas, its resistance decreases and the voltage is smaller, the V-voltage changed higher, so that the $\mathrm{V}+<\mathrm{V}-$. The LM393 outputs the low level, the light emitting diode conducts. The single chip microcomputer detects the low level, so as to start the alarm.
The detection circuit of the smoke and combustible gas is shown in Figure 4:

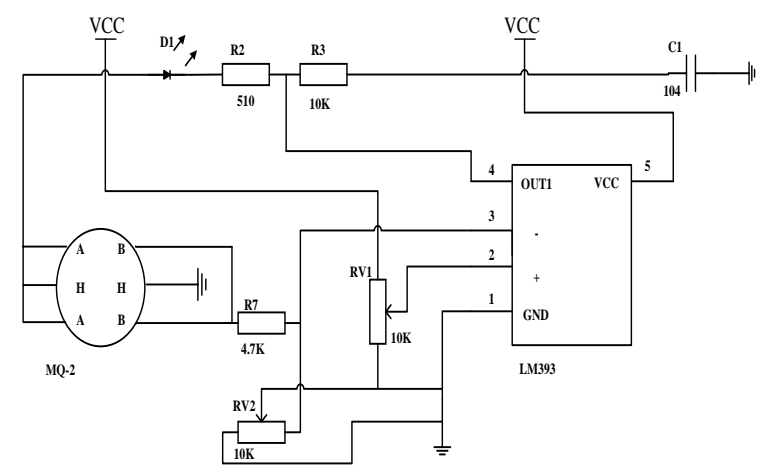

Figure 4. The circuit of the smoke and the combustible gas.

\section{3) Human infrared detection}

With the HC-SR501 module, the system detects the human body. When the human body passes through the infrared detection zone, the MCU outputs the trigger signal to the wireless module. When no one is unmanned, the MCU is used to detect the high level through the pull resistance[7].

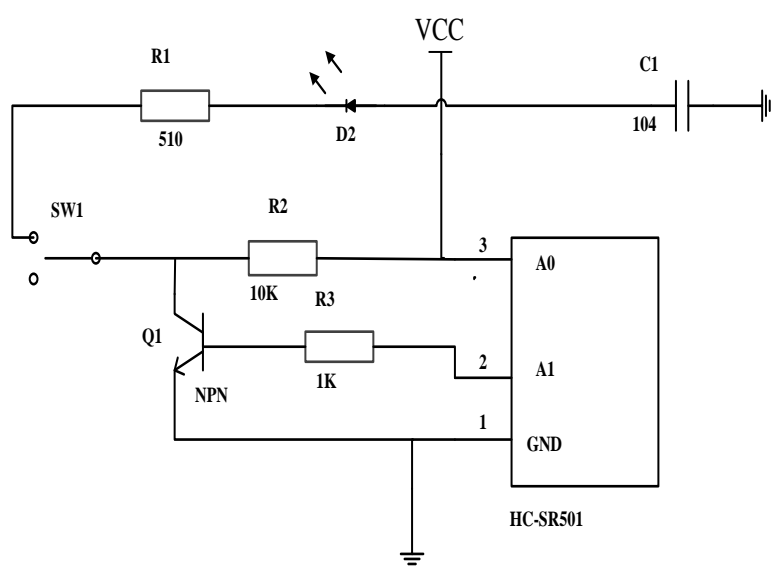

Figure 5. The infrared detection circuit.

HC-SR501 is the human body induction module. When it detects the human body, pin A1 outputs the high level. Through the current limiting resistor, Q1 turns on and the collector connects to the GND and then switched to the low level. At this time, the light emitting diode conducts and the LED lighting. The single chip microcomputer detects the low level and begins an alarm. In the circuit, the capacitance used as the filter, R2 is the pull-up resistor. When there is no person, the Q1 turns off. The single chip microcomputer can detect the high level by the pull-up resistor.

\section{SOFTWARE SYSTEM DESIGN}

\section{A. Main program design}

The software of this system is designed with a single chip system program as the main program, and the sensor module 
program is designed as the subroutine[8]. The main program flow is shown in Figure 6.

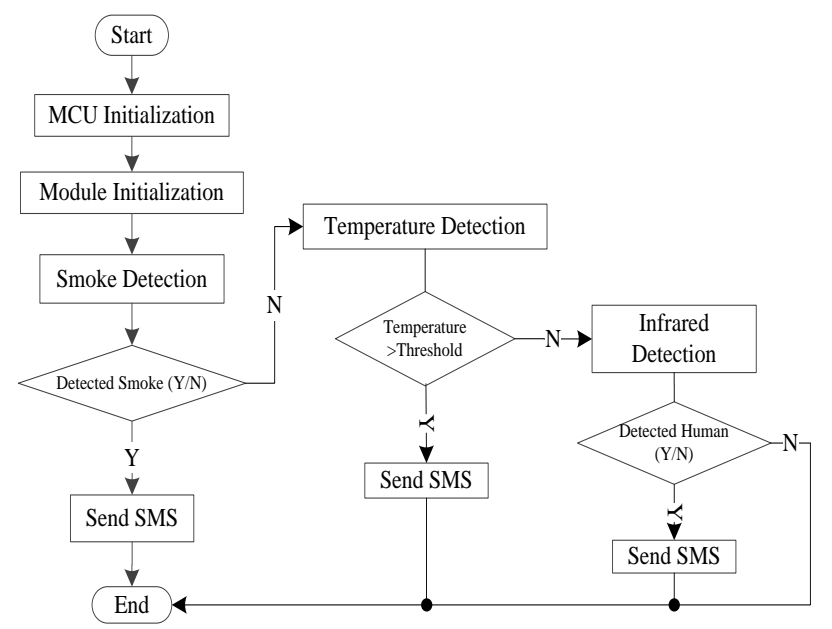

Figure 6. Main program of the systemm.

At first, the MCU initializes and then the temperature module, the human infrared module and the smoke alarm module are also initialized. If the system detects the smoke, sends the message to the mobile phone or determines whether the temperature exceeds the threshold value. If it exceeds it, send data to the user or judge the results of the human detected. If detects the human body sends the data to the mobile phone or not.

\section{B. GSM module program design}

The program design of this part is the design of serial communication of GSM[9]. First, port, baud rate, parity bit, data bit and so on are first set up, then the short message is sent by TC35 AT command. The flow of its program is shown in Figure 7.

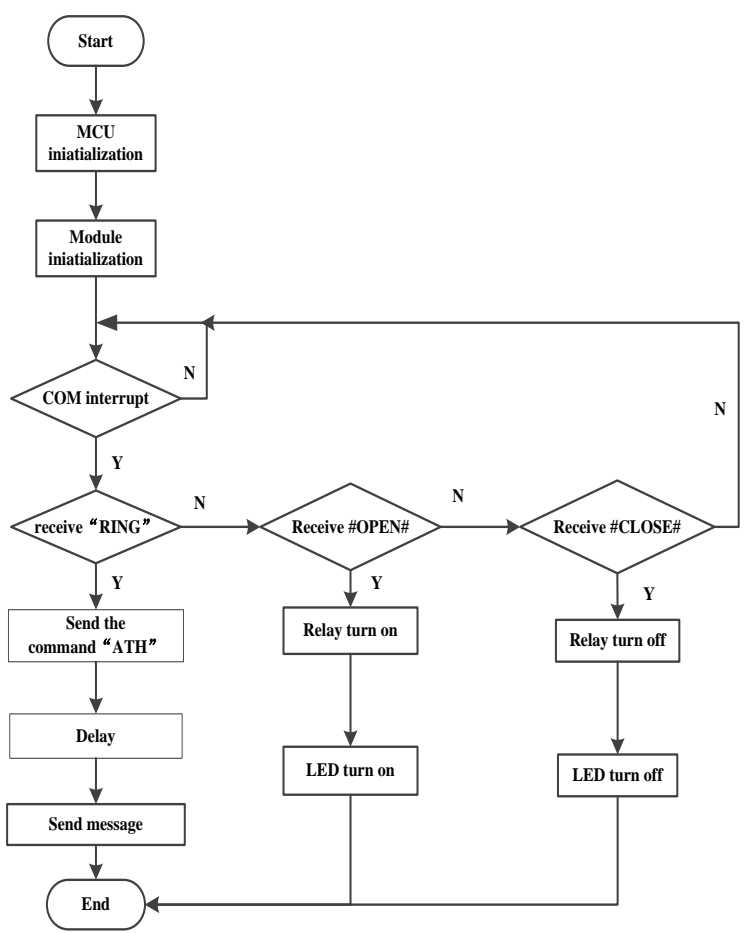

Figure 7. TC35 module program flow chart.

The program began, GSM module initialization, serial port to determine whether to interrupt if the serial interrupt, and then determine whether to receive the "RING" command, if the received command is sent on command, if not to receive orders and then to determine whether the received switch command, if the \#OPEN\# is received, the control relay is closed, LED lights, if received the \#CLOSE\# command, control relay off, LED lamp.

\section{TEST RESULT AND DATA ANALYSIS}

When the system detects the data exceeds the threshold, the GSM module begin works. It send SMS to the user or the main platform, which could transmit the data in time. In order to display the test results, uses the screen results of the mobile phone in Figure 8.

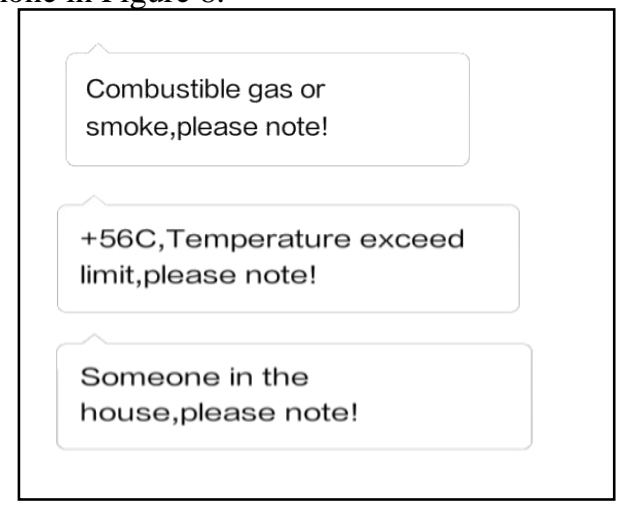

Figure 8. GSM sending SMS.

Users can send "OPEN" and "CLOSE" command to control the working status of the system. If the "OPEN" 
command is send, the LED turns on. When sending "CLOSE" command the LED turns off. It proved the GSM module could receive short message successfully. The test result is shown in Figure 9.

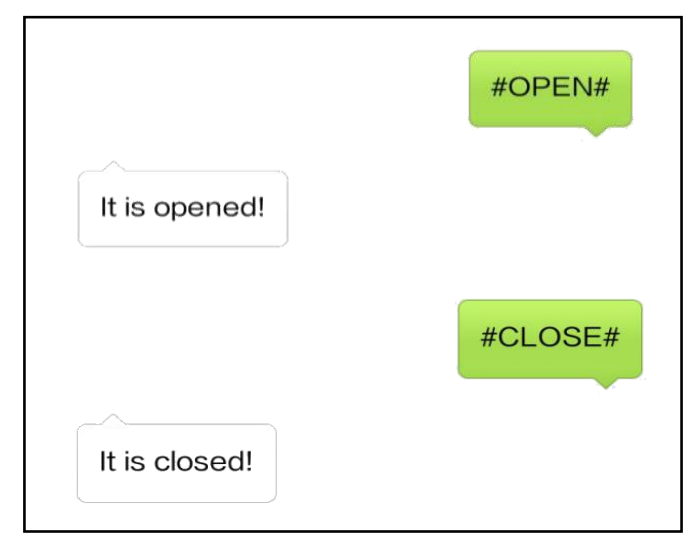

Figure 9. GSM receiving SMS

\section{CONCLUSIONS}

In this paper, the multi-function alarm system could detect the smoke or combustible gas, the infrared identification and the temperature by the multi-point sensor in the large stadium. The collected data transmitted by the mobile communication to the mobile phone or the main platform. The system is simple and cheap, it has various functions for the monitoring system conveniently.

\section{ACKNOWLEDGMENT}

This work was partly supported by Shaanxi Province Social Science Fund Project (NO.2016R022) of China and by the scientific research fund project of Shaanxi Provincial Department of Education (17JK1036).

\section{REFERENCES}

[1] F.Cappella, V.Caracciolo, and R.Cerulli, et al. "The calibration and the monitoring/alarm system", International Journal of Modern Physics A, vol. 31, Nov. 2016, pp.1-8, doi:org/10.1142/S0217751X16420045

[2] T.Hamaguchi, H.Sakashita, and H.Moritani, et al. "Method for Designing Alarm System Using DAEs, CE Matrices, and Preference Indices", Journal of Chemical Engineering of Japan, vol.50, Jun,2017, pp.439-444, doi:org/10.1252/jcej.16we365

[3] S.Romero-Brufau, B. W.Morlan, and M.Johnson, et al. "Evaluating Automated Rules for Rapid Response System Alarm Triggers in Medical and Surgical Patients”, Journal of Hospital Medicine, vol.23, Nov.2017, pp.217-223, doi: 10.12788/jhm.2712.

[4] Y.Jie, H.Zhu, and X.Cao. "One-Piece Triboelectric Nanosensor for Self-Triggered Alarm System and Latent Fingerprint Detection", Acs Nano, vol.11, Oct. 2016, pp.10366-10372, doi:10.1021/acsnano.6b06100.

[5] Y.Jiang, G.Li, and J.Wang . "Photoacoustic Compound Fire Alarm System for Detecting Particles and Carbon Monoxide in Smoke", Fire Technology, vol.52,May.2016, pp.1255-1269, doi:org/10.1007/s10694-015-0542-6.

[6] S.Fong, R.Wong, and A. V.Vasilakos. "Accelerated PSO Swarm Search Feature Selection for Data Stream Mining Big Data", IEEE Transactions on Services Computing, vol.9, Dec.2016, pp.33-45, doi:10.1109/TSC.2015.2439695.

[7] A. O.Gaca, P.Kudrin, and C.Colomer-Winter, et al. "From (p)ppGpp to (pp)pGpp: Characterization of Regulatory Effects of pGpp Synthesized by the Small Alarmone Synthetase of Enterococcus faecalis", Journal of Bacteriology, vol.197, Oct.2015, pp.2908.doi:10.1128/JB.00324-15.

[8] T.Jones, L.Glass, and S.Gandhi, et al. "Madagascar's Mangroves: Quantifying Nation-Wide and Ecosystem Specific Dynamics, and Detailed Contemporary Mapping of Distinct Ecosystems", Remote Sensing, vol.8,Feb.2016, pp.106,doi:10.3390/rs8020106.

[9] A. S.Crunchant, M.Egerer, and A.Loos, et al. "Automated face detection for occurrence and occupancy estimation in chimpanzees", American Journal of Primatology, vol.79,May.2017, pp.112.doi:10.1002/ajp.22627. 